\title{
Le competenze dello specialista in reumatologia: un documento del Consiglio Europeo di Reumatologia
}

\author{
Traduzione a cura di \\ Virginia D'Abrosca e Rosaria Irace, \\ specializzande in Reumatologia presso la Seconda Università di Napoli
}

\section{PREMESSA}

Il Consiglio Europeo di Reumatologia (CER) è l'organismo che rappresenta i reumatologi nell'ambito dell'UEMS (Union Europèenne des Médicins Spècialistes).

In accordo con lo statuto dell'UEMS, Il CER è costituito da membri scelti da organizzazioni di Reumatologi (ndr: comunemente uno in rappresentanza della Società scientifica, uno della Società Professionale) di ciascuno degli Stati membri della Comunità Europea e dei Paesi aderenti alla EFTA.

Nell' ambito degli obiettivi fondanti, il CER persegue la definizione di direttive comuni nel campo della formazione, allo scopo di assicurare i più alti livelli di assistenza medica reumatologica in ogni parte d'Europa.

Il CER ha recentemente pubblicato raccomandazioni sulle prestazioni che un servizio di reumatologia dovrebbe erogare al fine di fornire l'appropriata assistenza medica a pazienti con patologie muscolo-scheletriche ${ }^{1}$.

Il CER, inoltre, nel Dicembre 2006, ha rivisto ed approvato un Documento sulla formazione in Reumatologia nell' $\mathrm{UE}^{2}$.

Esso rappresenta un ulteriore passo in avanti verso l'armonizzazione della formazione degli specialisti reumatologi all'interno dell'Unione Europea.

Esso, infatti, fornisce un quadro di riferimento per l'elaborazione e l'individuazione dei parametri dei Curricula nazionali per la formazione specialistica in Reumatologia, costituendo una revisione di

Indirizzo per la corrispondenza:

European Rheumatology Board - Section

of UEMS- EULAR House - Zurich primaria importanza del Curriculum dello Specialista Reumatologo elaborato dall'UEMS nel 2003. Il presente documento è stato elaborato in aderenza allo Schema di Riferimento per le Competenze del Medico del CanMED 200533 (The Royal College of Physicians and Surgeons of Canada 20052007), utilizzando quali ulteriori fonti gli Elementi Essenziali del Curriculum dell'American College of Rheumatology ${ }^{4}$ e alcuni Curricula Nazionali Europei di Reumatologia5.

Il Prof. Reg Dennick, Assistente Dirigente del Dipartimento di Educazione Medica dell'Università di Nottingham ha fornito il suo contributo di esperto. Il presente documento è stato approvato all'unani-

\footnotetext{
${ }^{1}$ Woolf AD and The European Union of Medical Specialists Section of Rheumatology/European Board of Rheumatology. Health Care Services for those with musculoskeletal conditions: a rheumatology service. Recommendations of the European Union of Medical Specialists Section of Rheumatology/European Board of Rheumatology 2006. Ann Rheum Dis 2007; 66: 293-301.

${ }^{2}$ Da Silva JAP, Faarvang K-L, Bandilla K, Woolf AD on behalf of the UEMS Section and Board of Rheumatology. UEMS charter on training of rheumatologists in Europe. Ann Rheum Dis 2008. In Press.

${ }^{3}$ Frank JR., ed. 2005. The CanMEDS 2005 physician competency framework. Better standards. Better physicians. Better care. Ottawa: The Royal College of Physicians and Surgeons of Canada. http://rcpsc.medical.org/canmeds/CanMEDS2005/ CanMEDS2005_e.pdf

${ }^{4}$ American College of Rheumatology. Core Curriculum Outline for Rheumatology Fellowship Programs. A Competency-Based Guide to Curriculum Development. http://www.rheumatology.org/educ/training/CCO.pdf

${ }^{5}$ The Danish, Curriculum for Specialist Training in Internal Medicine: Rheumatology. National Board of Health. Danish Society for Rheumatology. January 2004. Edited in January 2005. The British, Specialty Training Curriculum For Rheumatology. Joint Royal Colleges of Physicians Training Board. May 2007 (http://www.uemsrheumatology.net; www.jrcptb.org.uk).
} 
mità da tutti i componenti del $\mathrm{CER}^{6}$, i quali si sono anche confrontati con i rappresentanti del Gruppo di Lavoro Permanente dei Giovani Medici dell'Unione Europea.

Esso dovrebbe essere tenuto in conto dalle Autorità dei singoli Stati insieme alla Guida alla Formazione dello stesso CER, che definisce le modalità per costruire e valutare il Curriculum dello Specialista in Reumatologia, nel rispetto delle esigenze nazionali.

\section{OBIETTIVI}

È noto che le condizioni in cui vengono praticate la medicina e la reumatologia, cosi come le relative regolamentazioni, possono variare fra i diversi paesi, e che rimarranno tali, e che la individuazione degli obiettivi, della struttura e dei contenuti del curriculum medico, rimangono nella sfera di competenza di autorità nazionali.

Cionondimeno, si ritiene che l'armonizzazione della formazione dello specialista in Europa sia essenziale per garantire standard comuni di assistenza e per promuovere la libera circolazione del medico specialista tra gli stati membri dell'UE.

In questa luce, il presente documento sulle linee guida di formazione dello specialista in Reumatologia, fornisce un'importante opportunità di migliorare gli standard di qualità delle prestazioni erogate a pazienti con patologie muscolo-scheletriche.

Il CER non ha l'intenzione né l'autorità per imporre ai singoli paesi una struttura, un contenuto o obiettivi definiti del curriculum in Reumatologia.

Questo documento, pertanto, vuole solo offrire alle autorità nazionali ed agli enti professionali impegnati nell'elaborazione dei curricula per la formazione dei reumatologi, un dettagliato schema di riferimento di competenze che devono essere acquisite al termine del percorso di formazione specialistica in Reumatologia in Europa.

Esso, inoltre, potrà essere utilizzato dagli specializzandi al fine di identificare i parametri utili a valutare il proprio standard di formazione e, eventualmente, quale fonte di ispirazione per promuovere un cambiamento in positivo, se necessario.

Le strategie didattiche adottabili per acquisire le competenze di Specialista in Reumatologia sono varie.

Ciascun paese, quindi, identificherà modalità di costruzione del curriculum in dipendenza delle pro- prie tradizioni e dalle risorse disponibili. Il CER ha, quindi, deciso di astenersi dalla individuazione di una qualunque struttura fissa del curriculum.

Tuttavia, si è ritenuto che l'armonizzazione fra i vari paesi e la promozione della qualità possano essere favorite dalla precisazione delle procedure da seguire nella definizione e nella elaborazione del curriculum.

È stato, quindi, prodotto un ulteriore documento che sottolinea le qualità ed i limiti dei differenti metodi di insegnamento e di valutazione: Guida per la Formazione del Consiglio Europeo di Reumatologia e che è disponibile in rete (http://www.uemsrheumatology.net/).

\section{Il quadro delle competenze. I ruoli del medico}

In aderenza allo Schema di Riferimento per le Competenze Mediche del CanMED 2005, che è stato scelto per la precisazione in esso contenuta del ruolo complesso ed in continua evoluzione che il medico deve svolgere nella medicina moderna, il medico, nella sua attività, svolge 7 ruoli o funzioni (Esperto, Comunicatore, Collaboratore, Manager/Leader, Difensore della salute, Studioso, Professionista), che costituiscono, nel presente documento, la struttura per l'organizzazione delle competenze, alcune delle quali come la comunicazione e la professionalità, precedentemente ignorate, devono essere pienamente riconosciute come fondamentali per la professione medica e non possono più essere lasciate alla determinazione individuale non sottoposta a verifica.

La scelta è ricaduta sullo Schema di riferimento per la Competenza Medica del CanMED $2005^{7}$ perché si è ritenuto che i parametri identificati da questo Organismo riflettano il passaggio dell'istruzione dalla definizione degli obiettivi in termini di nozioni e abilità a quella di competenze, intese come capacità di usare le nozioni, abilità e attitudini adeguate a risolvere problemi clinici in modo professionale, etico e competente al fine di

\footnotetext{
${ }^{6}$ Austria, Belgio, Croazia, Cipro, Repubblica Ceca, Danimarca, Estonia, Finlandia, Francia, Germania, Grecia, Ungheria, Islanda, Irlanda, Italia, Lussemburgo, Malta, Paesi Bassi, Norvegia, Polonia, Portogallo, Romania, Slovacchia, Slovenia, Spagna, Svezia, Svizzera, Turchia, United Kingdom.

${ }^{7}$ Frank JR. ed., 2005.The CanMEDS 2005 physician competency framework. Better standards. Better physicians. Better care. Ottawa: The Royal College of Physicians and Surgeons of Canada. http://rcpsc.medical.org/canmeds/CanMEDS2005/CanMEDS 2005_e.pdf
} 
raggiungere risultati ottimali per il paziente e per la società.

1 ruolo dell'Esperto continuerà, ovviamente, ad occupare un posto centrale nella formazione e nell'addestramento del medico, ma le competenze necessarie a svolgere quello del Comunicatore, del Collaboratore, del Manager/Leader, del Sostenitore della salute, dello Studioso e del Professionista dovrebbero essere chiaramente indicate nei programmi di formazione.

Il presente documento, quindi, si articola in 7 sezioni ciascuna delle quali inizia con la definizione del ruolo, e una descrizione più ampia della sue caratteristiche, con particolare riferimento a quelle del Reumatologo, e prosegue con la precisazione dei diversi aspetti da prendere in considerazione all'interno di tale ruolo specifico.

Sono inizialmente definite le competenze necessarie per svolgere ciascun ruolo in base ai livelli da raggiungere nel corso del processo formativo. Sono, quindi, descritte, in modo più dettagliato, le esigenze specifiche di formazione per l'acquisizione delle competenze stesse.

Sono, inoltre, suggeriti i metodi appropriati di insegnamento, apprendimento e di valutazione delle competenze relative a ciascun ruolo.

Una descrizione approfondita di questi metodi può essere trovata nella Guida per la Formazione del Consiglio Europeo di Reumatologia.

\section{L'esperto.}

\section{La capacità di prendere decisioni cliniche Definizione}

In qualità di Esperto, il reumatologo incorpora tutti i ruoli e esercita tutte le competenze elencate in questo documento, mettendo in pratica, nella sua attività di assistenza incentrata sul paziente, le conoscenze mediche, le abilità cliniche e le attitudini professionali acquisite. L'esperto è il ruolo medico centrale nella pratica reumatologica.

\section{Descrizione}

Il reumatologo possiede un insieme definito di nozioni, di capacità cliniche e procedurali e di attitudini professionali che sono dirette all'assistenza efficace dei pazienti con affezioni muscolo-scheletriche.

Egli svolge un'attività caratterizzata da una pratica clinica aggiornata, etica ed efficiente in rapporto alle risorse così come da una comunicazione efficace nel rapporto con i pazienti, con le altre figure professionali operanti nell' ambito dell' assistenza, e con la comunità.
Nell'esercizio del ruolo di Medico Esperto, egli utilizza le competenze del Comunicatore, del Collaboratore, del Manager/Leader, del Sostenitore della Salute, dello Studioso e del Professionista.

\section{Elementi}

- Conoscenze mediche di base

- Conoscenza approfondita delle malattie muscolo-scheletriche e dei problemi connessi ad esse.

- Raccogliere l'anamnesi ed eseguire l'esame obiettivo.

- Operare un ragionamento diagnostico.

- Operare una valutazione clinica.

- Prendere decisioni cliniche.

- Valutare il rapporto rischio-beneficio e l'economicità di un farmaco.

- Valutare l'impatto delle affezioni muscolo-scheletriche.

- Applicare una gestione adeguata delle evidenze.

- Abilità nelle competenze procedurali.

- Leadership nel gruppo.

- Conoscenza della Medicina basata sulle evidenze.

- Erogare una cura empatica.

- Integrare tutte le competenze per raggiungere la cura ottimale del paziente.

- Applicare principi etici per la cura del paziente.

\section{Competenze Chiave}

Entro la fine del percorso formativo un Reumatologo deve essere capace di...

- Dimostrare capacità diagnostiche, terapeutiche e di gestione del paziente con affezioni muscoloscheletriche e del tessuto connettivo, nell'ambito di un'assistenza etica ed efficace rispetto ai costi.

- Lavorare in un team multiprofessionale e multidisciplinare, riconoscendo i limiti delle proprie competenze.

- Valutare ed applicare le informazioni che sono importanti per la pratica clinica

- Fornire un valido supporto per lo sviluppo di servizi connessi alla prevenzione delle malattie, all'assistenza del paziente, all'educazione del paziente e dei suoi familiari, al supporto sociale, alla formazione medica e ad esprimere pareri medico-legali.

\section{Esigenze specifiche del processo formativo}

Al completamento della formazione, lo specializzando deve dimostrare di aver acquisito il seguente patrimonio di conoscenze specifiche, capacità e attitudini. 


\section{SEZIONE A REQUISITI DI CONOSCENZA (NDR SAPERE)}

\section{A. 1. Nozioni Generali}

Conoscenza operativa ${ }^{8}$, applicata alle patologie muscolo-scheletriche, di:

A. 1.1 Classificazione delle malattie muscolo-scheletriche.

A. 1.2 Metodi epidemiologici per lo studio delle malattie reumatiche.

A. 1.3 Statistica di base per le scienze mediche.

A. 1.4 Principi di medicina basata sulle evidenze.

A. 1.5 Conseguenze economiche, psicologiche e sociali delle malattie reumatiche.

A. 1.6 Ordinamento del proprio sistema sanitario locale, incluse l'assegnazione di risorse e le politiche sociali specifiche per le malattie muscolo-scheletriche.

\section{A. 2 Discipline di base}

Conoscenza operativa, applicata alle patologie muscolo-scheletriche, di:

A. 2.1 Anatomia e fisiologia dei tessuti muscoloscheletrici, tra cui struttura e funzione di ossa, articolazioni, tessuto connettivo, muscoli, tendini, nervi e vasi sanguigni, nel soggetto sano.

A. 2.2. Immunologia inclusa la struttura di base e la funzione degli organi linfoidi centrali e periferici, dei componenti cellulari e molecolari del sistema immunitario nel soggetto sano e nelle malattie muscolo-scheletriche.

A. 2.3. Fisiopatologia delle malattie muscolo-scheletriche (indispensabile per la comprensione dei meccanismi di malattia e la scelta della terapia) inclusi: biologia cellulare e molecolare, biomeccanica, fisiopatologia del dolore, genetica, meccanismi immuno-mediati (autoimmunità, immunocomplessi, graft versus host disease ecc.), ruolo di agenti infettivi, ruolo dell'invecchiamento.

A. 2.4. Farmacologia, compresi, tra gli altri, principi di base della gestione del farmaco, farmacologia dei farmaci utilizzati nelle malattie reumatiche e interazioni fra essi ed altri farmaci.

\section{A. 3 Discipline cliniche}

A. 3.1 Malattie e Problemi muscolo-scheletrici nell'adulto.

A. 3.1.1 Conoscenza approfondita e aggiornata del-

${ }^{8}$ Conoscenza operativa: informazione approfondita necessaria per un'esperta esecuzione di tutte le competenze elencate, nella prospettiva del reumatologo che opera nel proprio contesto nazionale. le malattie muscolo-scheletriche riportate nell'elenco allegato, incluse, per ciascuna malattia, l'epidemiologia, la genetica, la storia naturale, le manifestazioni cliniche, comprese quelle dei sottotipi clinici, i meccanismi patogenetici. Il grado di conoscenza richiesta rifletterà non solo la prevalenza, ma anche la potenziale gravità di ciascuna condizione nella pratica reumatologica nazionale del momento. A. 3.1.2 Conoscenza operativa delle malattie non muscolo-scheletriche coinvolte nella diagnosi differenziale o nella gestione del paziente con malattie muscolo-scheletriche quali le malattie cardiovascolari e renali, le distrofie muscolari, le interstiziopatie polmonari, il diabete, l'ipertensione, il glaucoma, le sindromi da ipercoagulabilità, le malattie infettive ecc.

A. 3.2 Malattie dell'apparato muscolo-scheletrico e del tessuto connettivo in età pediatrica.

A.3.2.1 Nei paesi europei, la reumatologia pediatrica è una specializzazione medica distinta, con competenze reumatologiche definite o condivise tra pediatri e reumatologi. Le presenti raccomandazioni sono finalizzate alla definizione delle competenze del Reumatologo, il quale è spesso responsabile dell'assistenza di pazienti colpiti da bambini da malattie muscolo-scheletriche, i quali ne continuano a soffrire nel corso dell'adolescenza fino all'età adulta e che, pertanto, deve essere preparato ad occuparsi di adolescenti ed ad affrontare le malattie pediatriche che continuano nell'età adulta e le loro conseguenze.

A. 3.2.2 Operare una diagnosi differenziale delle malattie elencate al paragrafo $n$. 15 dell'elenco sotto riportato, comprese le malattie non muscoloscheletriche che possono mimare condizioni muscolo-scheletriche nei bambini (paragrafo n. 16) A. 3.2.3 Conoscere i principi di gestione del bambino con malattie muscolo-scheletriche.

A. 3.2.4 Conoscere la storia naturale delle malattie muscolo-scheletriche pediatriche (paragrafo $\mathrm{n}$. 15) e delle loro complicanze maggiori (paragrafo n. 17).

A. 3.3 Indagini.

A. 3.3.1. Conoscenza del razionale biologico, dell'utilità, dei costi, dei limiti e dei principi interpretativi di tutte le indagini utilizzate per la regolare gestione di sindromi e malattie, incluse la conoscenza operativa delle metodiche utilizzate e le caratteristiche di ciascun test (sensibilità, specificità e valore predittivo).

A. 3.3.2. Le indagini da conoscere comprendono: - Test di laboratorio (compresi i test immunologici specialistici). 
- Metodiche di Imaging (incluse radiografie standard, tomografia computerizzata, risonanza magnetica, ecografia e indagini di medicina nucleare applicate allo studio di ossa, articolazioni, strutture periarticolari e vascolari).

- Tecniche di misurazione (densitometria ossea, studio quantitativo con ultrasuoni).

- Test neurofisiologici.

- Capillaroscopia.

- Artroscopia.

- Biopsie.

- Analisi del liquido sinoviale incluso lo studio con il microscopio a luce polarizzata.

\section{A. 4. Terapia}

A.4.1 Indicazioni/controindicazioni, gestione, costi, monitoraggio, effetti collaterali di farmaci e di tecniche (e.g. infiltrazioni), regolarmente utilizzati nel trattamento delle patologie muscolo-scheletriche.

A. 4.2 Conoscenza operativa delle indicazioni, dei rischi e dei limiti della terapia fisica e della riabilitazione, i.e. chinesiterapia (range di movimento, potenziamento, condizionamento, e stretching), riposo e immobilizzazione, idroterapia, cure termali, tecniche di protezione delle articolazioni, dispositivi adattativi e sostitutivi, calzature ortopediche, plantari.

A. 4.3 Conoscenza operativa dei metodi utilizzati per la prevenzione delle malattie muscolo-scheletriche comprese le problematiche connesse al lavoro, allo stile di vita, all' alimentazione e all'educazione del paziente.

A. 4.4 Capacità di un scegliere in modo appropriato quando inviare un paziente allo specialista della riabilitazione e al terapista del dolore.

A. 4.5 Capacità di comprendere gli aspetti psicosociali della malattia e della disabilità conseguente ed il loro impatto sul piano della gestione terapeutica, inclusi i fattori psicologici ed emotivi i.e. la sessualità, rapporti familiari e lavorativi, i problemi professionali, i costi e il monitoraggio della terapia.

A. 4.6 Conoscenza operativa delle indicazioni, della valutazione preoperatoria delle procedure chirurgiche impiegate nel trattamento delle malattie muscolo-scheletriche, nonché delle controindicazioni, delle complicanze, della gestione post -operatoria e dei risultati attesi.

A. 4.7. Medicina complementare: conoscenza operativa delle pratiche alternative, incluse la dieta, $\mathrm{i}$ supplementi alimentari, gli antimicrobici, l'agopuntura, la chiropratica, i farmaci topici, i rimedi omeopatici ecc.

\section{SEZIONE B CAPACITÀ CLINICHE (NDR SAPER FARE)}

Capacità di raccogliere ed interpretare le informazioni relative ad un paziente con affezioni muscolo-scheletrici (i.e. anamnesi, esame fisico, esami di laboratorio e strumentali) al fine di operare una diagnosi differenziale, valutare lo stato di salute globale, pianificare ulteriori valutazioni, organizzare e attuare un piano globale di gestione del paziente e valutarne l'effetto.

Al completamento della formazione lo specializzando dovrà dimostrare la capacità di:

B. 1. Raccogliere un'anamnesi, dal paziente o dai parenti, che sia pertinente, concisa, accurata e adeguata al problema, compresa la valutazione del problema dal punto di vista del paziente.

B. 2. Eseguire un esame fisico completo, compresa una valutazione dettagliata dell' apparato locomotore modulata ai problemi del paziente. L'anamnesi e l'esame fisico devono identificare le manifestazioni non-articolari, in particolare quelle con potenziali implicazioni per la diagnosi e/o la gestione delle malattie muscolo-scheletriche.

B. 3. Utilizzare, applicare ed interpretare le misure di attività, di stato funzionale, e di danno cumulativo appropriate per la malattia del paziente.

B. 4. Elaborare una diagnosi differenziale adeguata e programmare un piano diagnostico, che dimostri un uso ed un'interpretazione razionale delle indagini pertinenti, inclusa la valutazione di un rapporto costo-efficacia vantaggioso.

B. 5. Analizzare e interpretare i dati clinici, di laboratorio e di imaging emersi dai procedimenti sopra elencati per definire la/le diagnosi più probabile/i e operare una completa valutazione dello stato del paziente.

B. 6. Sviluppare un piano di gestione adeguato, basato su una informazione scientifica aggiornata, nonché su una valutazione clinica, che consideri i costi, le scelte e le condizioni del paziente, ivi compresa la dimostrazione della capacità di utilizzare farmaci e altre opzioni terapeutiche, promuovere l'educazione ed il sostegno del paziente e dei suoi familiari, contemperare l'impiego di cure preventive e l'integrazione delle competenze di altri professionisti della salute. Il nuovo reumatologo dovrà dimostrare di saper utilizzare in modo appropriato i farmaci in circostanze particolari come nei bambini, in gravidanza, nell' allattamento, nell'insufficienza renale e in altre condizioni.

B. 7. Riconoscere, valutare e gestire adeguatamente le emergenze reumatologiche, come la crisi renale 
sclerodermica, l'ipertensione arteriosa polmonare, la lussazione atlanto-assiale, la sindrome da anticorpi antifosfolipidi severa, l'arterite temporale ed altre.

B. 8. Progettare un adeguato piano di follow-up, che comprenda la valutazione della risposta al trattamento e il riconoscimento degli eventi avversi.

B. 9. Dimostrare la capacità di una reale, appropriata e tempestiva cooperazione con altri professionisti della salute in aderenza alle necessità di un'assistenza ottimale al paziente.

B. 10. Competenze tecniche ${ }^{9}$.

Al completamento della formazione lo specializzando dovrà dimostrare la capacità di:

B.10.1. Aspirare liquido sinoviale da articolazioni e borse.

B.10.2. Infiltrare articolazioni e tessuti molli.

B.10.3. Eseguire un'analisi del liquido sinoviale, inclusa la ricerca di cristalli con microscopio a luce polarizzata a luce polarizzata.

B.10.4. Interpretare reperti di imaging dell'apparato locomotore comprese una scintigrafia ossea e una densitometria ossea.

\section{Competenze facoltative}

B.10.5. L'acquisizione delle competenze sottoelencate può essere oggetto di regolamentazione a livello nazionale:

- Valutare a fini diagnostici biopsie di tessuti e organi (sinovia, cute, grasso sottocutaneo, ghiandole salivari minori, ossa, muscoli, nervi, rene, arteria temporale, ecc.).

- Eseguire una densitometria ossea.

- Eseguire un'ecografia muscolo-scheletrica.

- Eseguire una capillaroscopia.

- Eseguire un'elettromiografia.

- Eseguire un'artroscopia.

- Eseguire tecniche di infiltrazione eco guidata.

- Eseguire sinoviortesi radioattiva o chimica.

- Altro.

B. 11. Capacità di utilizzare le competenze e le attitudini sottoelencate, al fine di assicurare i più alti standard di assistenza per i pazienti ed un prezioso contributo allo sviluppo professionale proprio e degli altri, incluse la capacità di:

- Fornire assistenza compassionevole e umana.

- Lavorare in un team multidisciplinare e multi professionale.

${ }^{9}$ Il curriculum nazionale dovrebbe chiarire quali competenze tecniche sono considerate obbligatorie e che livello di prestazione deve essere dimostrata al completamento della formazione.
- Fornire tempestivamente valutazioni ben documentate e raccomandazioni in forma scritta e / o verbale.

- Valutare il grado di disabilità nell'ambito della sicurezza sul lavoro e della determinazione dell'indennizzo spettante ai lavoratori ecc.

- Ottenere, valutare criticamente e applicare le informazioni da ogni fonte per mantenere i più alti standard di valutazione, cura e gestione del paziente.

- Mostrare comprensione dei propri limiti di competenza attraverso l'autovalutazione.

- Individuare e rispondere adeguatamente alle questioni etiche rilevanti nella pratica clinica reumatologica.

- Dimostrare una competenza medica in situazioni diverse da quelle che coinvolgono l'assistenza diretta del paziente (ad esempio presentazioni di casi clinici, didattica, educazione del paziente e del medico di medicina generale, espressione di pareri medico-legali).

\section{Metodi di insegnamento ed apprendimento}

\section{A. Sapere}

I metodi e le risorse per acquisire il bagaglio raccomandato di conoscenze includono anche, ma non si limitano a:

- Letture indipendenti: trattati e manuali, riviste, internet.

- Partecipazione a convegni, conferenze o dibattiti.

- Partecipazione a presentazioni di casi clinici.

- Partecipazione a Corsi monotematici.

- Partecipazione a sedute di laboratorio.

- Coinvolgimento nella didattica.

- Revisione critica della letteratura - journal clubs, ecc.

\section{B. Saper fare}

La partecipazione attiva nella cura del paziente, sia con pazienti ambulatoriali che con pazienti ricoverati, è il pilastro centrale per l'acquisizione delle competenze.

Tali esperienze devono essere debitamente controllate in modo che il tirocinante abbia l'opportunità di seguire l'esempio del clinico specialista, partecipare alla gestione dei problemi e ricevere un riscontro adeguato e costruttivo. Sono funzionali a tale obiettivo:

- Incontri didattici: convegni, conferenze, dibattiti.

- Esperienza clinica in un contesto clinico sorvegliato e guidato.

- Discussioni interattive su casi clinici. 
- Letture indipendenti - trattati e manuali, articoli di riviste, e ricerche su internet.

- Preparazione di un portfolio dell'assistenza al paziente.

- Presentazione di casi clinici.

- Review di casi clinici basate su internet.

\section{Metodi di valutazione}

\section{A. Sapere}

- Valutazione delle capacità - con riferimento, in particolare, al patrimonio di conoscenze mediche.

- Valutazione da parte di Commissioni di Docenti.

- Esami formali orale o scritto.

- Valutazione delle prestazioni cliniche dello specializzando da parte del tutore.

\section{B. Saper fare}

- Valutazione periodica e riscontro del livello di formazione

- Valutazione delle capacità mediche- con riferimento, in particolare, all' assistenza al paziente.

- Valutazione da parte di Commissioni di Docenti.

- Revisione di documenti per la cura del paziente, la prescrizione dei farmaci, o l'elenco degli outcomes.

- Presentazioni ad un pubblico di colleghi e di profani.

- Partecipazione a progetti individuali o di gruppo di miglioramento della qualità.

- Esame pratico formale.

- Esercizio di valutazione clinica.

- Osservazione diretta delle abilità pratiche.

- Esame obiettivo clinico strutturato.

- Valutazioni a $360^{\circ}$.

- Revisione del bagaglio di conoscenze.

\section{PER APPROFONDIMENTI}

1. Guida alla formazione dell' "European Board of Rheumatology" (http://www.uems-rheumatology. net/).

2. Moore DE, Pennington FC, Practice-based learning and improvement, J Cont Educ Health Prof 2003; 23: S73-80.

3. Epstein RM, Mindful practice, JAMA, 1999; 282: 833-9.

4. "Advancing Education in Practice-Based Learning and Improvement". An educational resource developed by the ACGME to aid program directors in teaching and assessing PBLI located at www. acgme.org/outcome/implement/complete_PBLIBooklet.pdf

\section{IL COMUNICATORE}

\section{Definizione}

In qualità di Comunicatore, il reumatologo deve favorire l'instaurazione di un rapporto medico-paziente proficuo, che contempli una comunicazione bidirezionale quale quella che si realizza nel corso di affezioni spesso croniche.

\section{Descrizione}

Il reumatologo deve favorire e alimentare la comunicazione di scelte terapeutiche incentrate sul paziente attraverso la condivisione del processo decisionale e l'instaurazione di efficaci interazioni dinamiche con i pazienti, i familiari, altri operatori sanitari, altri professionisti e altre importanti figure implicate nel processo stesso. Le competenze di questo ruolo sono essenziali per instaurare un rapporto di fiducia, comunicare la diagnosi, fornire informazioni, promuovere la comprensione reciproca e favorire un piano condiviso di cura. La riservatezza e l'etica devono essere rispettate.

L'applicazione di queste capacità comunicative e la natura del rapporto medico-paziente variano in base ai livelli culturali, alle condizioni e alle esigenze di informazione del paziente. Ostacoli potenziali derivanti da differenze nel lessico e nel livello culturale devono essere individuati.

\section{Elementi}

- Approccio centrato sul paziente.

- Empatia, armonia, comprensione reciproca.

- Competenze relazionali nei rapporti con altri.

- Ascolto.

- Uso competente della comunicazione verbale e non-verbale.

- Rispetto per la diversità.

- Processo interattivo.

- Ricavare e sintetizzare l'informazione .

- Attenzione agli aspetti psicosociali della malattia.

- Comunicazione efficace delle informazione orali e scritte.

- Condivisione dei processi decisionali.

- Rapporto di fiducia ed etica nella relazione medico-paziente.

- Relazioni costruttive con il paziente, i familiari e gli altri operatori sanitari.

- Comprensione, affidabilità ed integrità.

- Flessibilità nell'applicazione delle capacità.

- Efficienza, precisione.

- Modulare le cattive notizie.

- Affrontare i problemi connessi con la morte. 
- Comunicare errori o eventi avversi.

- Consenso informato.

- Valutare le capacità.

- Fornire la documentazione necessaria.

- Comunicazione pubblica e attraverso i media, se necessaria.

\section{Competenze chiave}

Entro la fine del percorso formativo un reumatologo deve essere capace di...

1. Sviluppare una buona interazione con i pazienti, i loro familiari e gli altri operatori sanitari con empatia, fiducia reciproca sul piano terapeutico ed etico.

2. Registrare, selezionare, documentare e sintetizzare accuratamente le informazioni rilevanti e il punto di vista dei pazienti, dei loro familiari, dei colleghi e delle altre figure professionali.

3. Comunicare informazioni e chiarificazioni, in modo adeguato e comprensibile, sulle condizioni del paziente a questi, ai loro familiari, ai colleghi e alle altre figure professionali.

4. Proporre e negoziare un'intesa comune sulle aspettative, i problemi ed i progetti con i pazienti, i familiari, i colleghi e gli altri professionisti, allo scopo di definire un piano condiviso di assistenza.

5. Trasmettere un'informazione accurata ed efficace del problema medico verbalmente o per iscritto.

\section{Esigenze specifiche del processo formativo}

Per acquisire e dimostrare di aver acquisito le competenze sopra elencate, lo specializzando deve, al completamento della formazione, aver acquisito le seguenti nozioni, capacità e attitudini specifiche:

1. Sviluppo di una buona interazione con empatia, fiducia reciproca sul piano terapeutico ed etico con i pazienti, gli accompagnatori e le famiglie. 1.1. Essere un buon comunicatore. Il reumatologo deve riconoscere che questa è un'abilità clinica centrale nella sua pratica e deve sforzarsi di impadronirsene. Deve dimostrare di aver capito che una comunicazione medico-paziente efficace può favorire la soddisfazione e l'adesione del paziente al piano terapeutico e migliorare i risultati clinici.

1.2. Instaurare, per quanto concerne la terapia, relazioni positive con i pazienti e i loro familiari, che siano caratterizzate da comprensione, fiducia, rispetto, correttezza ed empatia reciproca.

1.3. Rispettare la riservatezza, la privacy e l'autonomia del paziente.
1.4. Avere una buona capacità di consulente, essendo capace di agevolare efficacemente un incontro clinico strutturato, di ascoltare realmente e di recepire e rispondere a messaggi non espressi verbalmente.

2. Registrare, selezionare, documentare e sintetizzare le informazioni e le prospettive dei pazienti, dei loro familiari, dei colleghi e delle altre figure professionali.

2.1. Raccogliere informazioni sulle condizioni cliniche, ma anche sulle convinzioni, le preoccupazioni, le aspettative e le esperienze di malattia del paziente.

2.2. Selezionare, valutare e documentare accuratamente le informazioni pertinenti così da trasmettere le informazioni in modo affidabile ad altri.

2.3. Ricercare e sintetizzare le informazioni pertinenti da altre fonti, come la famiglia del paziente, gli operatori sanitari e le altre figure professionali. 3. Trasmettere informazioni e chiarificazioni riguardanti il paziente in modo adeguato e comprensibile per i pazienti e i familiari, i colleghi e le altre figure professionali.

3.1. Trasmettere le informazioni al paziente ed ai familiari, ai colleghi e alle altre figure professionali in modo umano, riconoscendo le loro necessità e in modo tale da essere comprensibili, ed incoraggiare la discussione, la partecipazione al progetto decisionale e l'adesione al programma.

4. Proporre e negoziare un'intesa comune sulle indagini, i problemi e i progetti con i pazienti ed i loro familiari, i colleghi e gli altri professionisti per lo sviluppo di un piano condiviso di assistenza.

4.1. Individuare e analizzare efficacemente i problemi provenienti da un incontro col paziente, tra cui l'ambiente, le reazioni, le preoccupazioni e le preferenze del paziente.

4.2. Rispettare la diversità e le differenze, comprendendo, ma non limitandosi all'impatto che possono avere l'influenza del genere, del livello culturale e delle convinzioni religiose sul prendere decisioni.

4.3. Incoraggiare la discussione, le domande e l'interazione in ogni incontro.

4.4. Coinvolgere il paziente, i familiari e il personale sanitario in un progetto decisionale condiviso per sviluppare un programma di assistenza.

4.5. Affrontare efficacemente i problemi impegnativi legati alla comunicazione con il supporto dei soggetti con affezioni muscolo-scheletriche croniche.

4.6. Affrontare efficacemente problemi importanti 
di comunicazione, quali ottenere il consenso informato, comunicare cattive notizie, e affrontare reazioni di rabbia o confusione e malintesi.

5. Trasmettere informazioni accurate ed efficaci su problemi medici sia verbalmente sia per iscritto.

5.1. Archiviare relazioni chiare, accurate ed appropriate (in forma scritta o elettronica) degli incontri e dei programmi clinici.

5.2. Presentare efficacemente resoconti verbali degli incontri e dei programmi clinici.

5.3. Preparare resoconti per i datori di lavoro e gli enti.

5.4. Identificare i punti critici propri della presentazione delle informazioni su problemi medici al pubblico, alle compagnie d'assicurazione o ai mezzi d'informazione.

\section{Metodi d'insegnamento e di apprendimento}

I metodi e le risorse che possono contribuire all'acquisizione di queste competenze includono, ma non si limitano a:

- Apprendimento attraverso l'esperienza. L'apprendimento mediante collaborazione attiva con docenti-professionisti che siano esempio di pratiche corrette e che adottino attivamente un ruolo tutoriale nei confronti dello specializzando rappresenta probabilmente, in questo settore, il modo più efficace di promuovere la competenza.

- Discussioni di gruppo basate su casi clinici.

- Interpretazione del ruolo di comunicatore.

- Valutazione da parte di tutor di azioni visionate dal vivo o videoregistrate.

- Collaborazioni con associazioni di malati e associazioni pubbliche.

- Colloqui con i coniugi.

\section{Metodi di valutazione}

- Valutazione e riscontro regolari del processo formativo

- Valutazione di incontri videoregistrati

- Valutazione a $360^{\circ}$

- Valutazione di relazioni cliniche

- Revisione degli archivi clinici

\section{PER APPROFONDIMENTI}

1. Guida alla formazione dell'European Board of Rheumatology (http://www.uems-rheumatology. net/).

2. Moore DE, Pennington FC, Practice-based learning and improvement, J Cont Educ Health Prof 2003; 23: S73-80.
3. Epstein RM, Mindful practice, JAMA, 1999; 282: 833-9.

4. "Advancing Education in Practice-Based Learning and Improvement". An educational resource developed by the ACGME to aid program directors in teaching and assessing PBLI located at www.acgme. org/outcome/implement/complete_PBLIBooklet.pdf

\section{IL COLLABORATORE}

\section{Definizione}

In qualità di Collaboratore, il reumatologo lavora efficacemente all'interno di una squadra di operatori della salute e dell'assistenza sociale per raggiungere la cura ottimale del paziente.

\section{Descrizione}

Il reumatologo lavora in collaborazione con altre figure coinvolte nell'assistenza a singoli pazienti o a specifici gruppi di pazienti.

Nella moderna assistenza sanitaria la squadra comprende non solo un gruppo multidisciplinare di professionisti che lavorano a stretto contatto in una sede unica, come ad esempio un team di reparto, ma anche un gruppo esteso di operatori, dislocato in più sedi, con prospettive e competenze diverse.

$\grave{E}$, quindi, essenziale che il reumatologo sia in grado di collaborare efficacemente con i pazienti, con i familiari e con un gruppo inter-professionale di specialisti per fornire livelli ottimali di assistenza, informazione ed educazione del paziente.

\section{Elementi}

- Assistenza, cultura e ambiente basato sulla collaborazione.

- Condivisione delle conoscenze e delle informazioni.

- Rispetto per gli altri medici e gli altri membri del team di assistenza sanitaria.

- Rispetto per la diversità.

- Dinamica di squadra.

- Direttive basata sulle necessità del paziente.

- Processo decisionale condiviso.

- Capacità di delegare.

- Team efficace.

- Trattativa costruttiva.

- Risoluzione, gestione e prevenzione dei conflitti.

- Strutture organizzative che facilitino la collaborazione.

- Responsabilità e ruoli chiari. 
- Riconoscere il proprio ruolo ed i propri limiti.

- Consultazione efficace per quanto riguarda le dinamiche di collaborazione.

- Assistenza di base efficace.

- Collaborazione con organizzazioni della comunità.

- Scambi di informazioni.

- Assistenza sanitaria inter-professionale.

- Assistenza sanitaria multiprofessionale.

- Assicuratori nel campo della salute.

- Imparare insieme.

- Rapporti con operatori di altro sesso.

\section{Competenze chiave}

Entro la fine del percorso formativo un reumatologo deve essere capace di...

1. Partecipare in modo efficace e appropriato ad un team di assistenza sanitaria multiprofessionale e multidisciplinare.

2. Lavorare efficacemente con altri operatori sanitari e con associazioni di pazienti per affrontare e risolvere i problemi dell" assistenza.

3. Collaborare con associazioni di pazienti affetti da malattie muscolo-scheletriche.

\section{Esigenze specifiche del processo formativo}

Per acquisire e dimostrare le competenze suddette, lo specializzando, al completamento della formazione, deve acquisire le seguenti nozioni e possedere le seguenti capacità e attitudini specifiche.

1. Partecipare in modo efficace e appropriato ad un team di assistenza sanitaria multiprofessionale e multidisciplinare.

1.1. Conoscere, capire e rispettare i ruoli e le responsabilità e le dinamiche degli altri professionisti all'interno di un team di assistenza multiprofessionale e multidisciplinare.

1.2. Dimostrare un atteggiamento rispettoso verso gli altri colleghi ed gli altri membri del team interprofessionale.

1.3. Lavorare con gli altri componenti del team per pianificare, valutare e fornire un' assistenza multidisciplinare integrata a pazienti singoli (o a gruppi di pazienti), riconoscendo il forte impatto delle malattie muscolo-scheletriche sul paziente, sulle persone che lo assistono e sui suoi familiari.

1.4. Quando necessario, lavorare con gli altri componenti del team per valutare, pianificare, fornire e revisionare i rispettivi compiti, come per esempio l'attività di ricerca, il lavoro di formazione, la revisione dei programmi o le responsabilità amministrative.
1.5. Partecipare attivamente agli incontri del team multidisciplinare.

1.6. Rispettare l'etica del team, compresa la riservatezza, l'assegnazione delle risorse e la professionalità, lavorando in collaborazione con gli altri professionisti per prevenire i conflitti.

1.7. Se necessario, dimostrare un ruolo di leadership nella squadra.

2. Lavorare efficacemente con gli altri operatori sanitari e le associazioni per affrontare e risolvere i problemi riguardanti l'assistenza del paziente.

2.1. Dimostrare la conoscenza delle normative e delle prassi in materia di accesso alle cure adeguate. 2.2. Essere in grado di elaborare tesi basate sulle evidenze e sulle pratiche migliori per facilitare le trattative con enti regolatori.

2.3. Impiegare una negoziazione collaborativa per risolvere i conflitti.

2.4. Rispettare le differenze, le incomprensioni e i limiti degli altri professionisti.

2.5. Riconoscere le proprie differenze, incomprensioni e i limiti che possono contribuire alle tensioni inter-professionali.

2.6. Riflettere sulla funzione di un team inter-professionale e sul proprio contributo alla sua efficienza.

3. Collaborare con le associazioni di pazienti di malattie muscolo-scheletriche.

3.1. Conoscere le associazioni che sostengono i pazienti con affezioni muscolo-scheletriche, ciò che offrono i potenziali vantaggi di una proficua collaborazione con esse.

\section{Metodi d'insegnamento e di apprendimento}

I metodi e le risorse che possono contribuire all'acquisizione di queste competenze includono, ma non si limitano a:

- Apprendimento attraverso l'esperienza in Dipartimenti e con professionisti che siano esempio di buona prassi in questi settori.

- Discussioni di gruppo basate su casi clinici.

- Prestazione sotto supervisione seguita da una valutazione.

- Lavorare con organizzazioni di malati e associazioni pubbliche.

\section{Metodi di valutazione}

- Valutazione e riscontro regolari del processo formativo.

- Valutazione di incontri con il paziente.

- Valutazione a $360^{\circ}$.

- Revisione di resoconti clinici. 


\section{PER APPROFONDIMENTI}

1. Guida alla formazione dell'European Board of Rheumatology (http://www.uems-rheumatology.net/).

\section{IL MANAGER/ IL TEAM LEADER}

\section{Definizione}

In qualità di Manager/Capogruppo, il reumatologo deve essere preparato a partecipare attivamente all'organizzazione dell' assistenza sanitaria, facendosi promotore di ipotesi sostenibili e prendendo decisioni sull'allocazione delle risorse in un'ottica di efficienza del sistema sanitario.

\section{Descrizione}

Il reumatologo interagisce con il proprio ambiente di lavoro come individuo, come membro di un team e come esponente del sistema sanitario locale, regionale o nazionale.

Il reumatologo funge da Manager che sovraintende alle attività di un team multidisciplinare, all'allocazione di risorse e all'assegnazione di compiti organizzativi (ad esempio scelte di metodi e di politiche di assistenza), tenendo conto di ogni singolo componente.

È, quindi, richiesta al reumatologo la capacità di individuare le priorità, collaborare efficacemente con i colleghi e svolgere il proprio ruolo operando sistematicamente scelte sull'allocazione delle scarse risorse disponibili per l'assistenza sanitaria.

\section{Elementi}

- Organizzazione,struttura e finanziamento del sistema sanitario.

- Budget e finanziamento.

- Definizione delle priorità.

- Gestione delle attività sostenibili.

- Risorse della salute.

- Amministrazione.

- Opzioni per le retribuzioni dei medici.

- Trattative.

- Crescita professionale.

- Uso di strumenti tecnologici per i servizi sanitari.

- Costruzione di un servizio di Reumatologia.

- Garanzia di qualità e di miglioramento.

- Leadership.

- Supervisione.

- Collaborazione nelle decisioni.

- Incontri e riunioni.

- Cambiamento di modalità di gestione.

- Principi di giustizia e di efficienza nella riparti- zione delle risorse sanitarie per la cura ottimale dei pazienti.

- Gestione del tempo.

\section{Competenze chiave}

Il reumatologo deve essere in grado di...

1. Partecipare in modo efficace alle attività che contribuiscono alla efficienza dell'organizzazione dei sistemi sanitari.

2. Gestire l'attività e la carriera degli operatori sanitari in modo efficace.

3. Destinare in maniera corretta le risorse sanitarie nell'interesse dei pazienti e della comunità.

4. Essere impegnato in ruoli amministrativi e di gestione.

\section{Esigenze specifiche del processo formativo}

Queste competenze non possono essere pienamente acquisite durante il corso di Specializzazione, ma devono essere maturate attraverso un continuo sviluppo professionale.

I programmi di formazione specialistica devono, tuttavia, assicurare l'acquisizione delle competenze di base, e verificare che tali competenze siano state acquisite.

Al completamento della formazione, il reumatologo deve dimostrare di possedere le seguenti conoscenze, abilità e attitudini:

1. Partecipare in modo efficace alle attività di organizzazione di sistemi sanitari.

1.1. Lavorare in modo collaborativo con altri a progetti propri.

1.2. Comprendere e supportare i principi di valutazione e di miglioramento del processo di qualità, come lo sviluppo e l'applicazione di linee guida e raccomandazioni.

1.3. Conoscere la struttura e la funzione del sistema sanitario, incluso il ruolo dei medici, e la posizione della reumatologia nel suo contesto.

1.4. Conoscere i principi e saper analizzare i principali problemi di finanziamento delle prestazioni sanitarie.

2. Gestire la propria attività e la propria carriera in modo efficace.

2.1. Definire le priorità, il tempo da dedicare alla cura del paziente ed altre esigenze pratiche, al di fuori delle attività personali.

2.2. Descrivere i principi di gestione della pratica clinica, comprese la gestione delle risorse finanziarie e risorse umane.

2.3. Essere capace di di discutere e progettare lo sviluppo di servizi sanitari e le modalità di miglioramento delle pratiche professionali. 
2.4. Impiegare strumenti di informazione in modo appropriato per la cura dei pazienti.

3 . Valutare e destinare criticamente le limitate risorse sanitarie in modo adeguato nell'interesse dei pazienti e della comunità.

3.1. Dimostrare un'adeguata considerazione del rapporto costo-efficacia delle procedure impiegate nella cura del paziente.

3.2. Riconoscere l'importanza della corretta assegnazione di risorse sanitarie, bilanciando l'efficacia e l'efficienza con l'assistenza ottimale per il paziente.

3.3. Applicare le evidenze e processi di verifica del giusto costo delle cure prestate.

4. Essere impegnati in ruoli amministrativi e gestionali, a seconda dei casi.

4.1. Partecipare in modo efficace a riunioni sulla gestione.

4.2. Dimostrare capacità di leadership adeguate, compresa la supervisione degli specializzandi.

4.3. Dimostrare una buona conoscenza operativa della programmazione e dell'amministrazione dell'assistenza sanitaria (ad esempio: orari di lavoro, struttura e funzione di un dipartimento, rapporti e contabilità, ecc.).

\section{Metodi di insegnamento ed apprendimento}

I metodi e le risorse che possono contribuire alla acquisizione di tali competenze includono, tra l'altro:

- Esperienze di apprendimento in Dipartimenti che siano esempio di eccellenza nel proprio campo.

- Discussioni di gruppo basate su casi clinici.

- Corsi dedicati a metodi e principi di gestione.

\section{Metodi di valutazione}

- Valutazione e riscontro periodico della formazione.

- Cartelle cliniche.

- Valutazione a $360^{\circ}$.

- Valutazioni del livello di ciascuna competenza specifica, seguendo dei criteri di valutazione predefiniti.

\section{PER APPROFONDIMENTI}

1. European Board of Rheumatology Educational Guide (http://www.uems-rheumatology.net/).

2. Nolan T. Understanding medical systems. Ann Intern Med 1998; 128: 293-8.

3. Macones GA, Goldie SJ, Peipert JF. Cost-effective analysis: an introductory guide for clinicians. Obstet Gynecol Surv 1999; 54: 663-72.
4. Systems-based practice: to learn about and improve the system. ACGME Bulletin, November, 2004. www.acgme.org

\section{IL DIFENSORE DELLA SALUTE}

\section{Definizione}

In qualità di Difensore della Salute, il reumatologo deve usare responsabilmente la propria esperienza per migliorare lo stato di salute e il benessere dei singoli pazienti, della comunità e della popolazione, con particolare riguardo alle patologie dell' apparato locomotore e del tessuto connettivo.

\section{Descrizione}

Il reumatologo dovrebbe essere cosciente e accettare il proprio dovere di promuovere la salute dei suoi pazienti e della società. Egli conosce l'importanza del proprio ruolo per il singolo paziente e per la collettività.

Il paziente ha bisogno che il medico lo assista nel rapporto con le strutture del sistema sanitario e nell'accesso a strutture appropriate in modo tempestivo.

La collettività richiede al reumatologo una speciale competenza nell'individuazione dei rischi sanitari e delle soluzioni per quanto riguarda le problematiche relative all'apparato locomotore e un fattivo contributo alla corretta ripartizione delle risorse sanitarie.

A tal proposito la difesa della salute implica uno sforzo di cambiamento sia delle procedure che delle politiche sanitarie in generale. Ciò si traduce in azioni individuali e collettive dei medici.

\section{Elementi}

- Sostegno dello stato di salute del singolo paziente e della comunità.

- Promozione della salute e prevenzione delle malattie.

- Indicatori di salute (psicologici, biologici, sociali, culturali, giuridici ed economici).

- Peso socio-economico individuale e collettivo delle malattie muscolo-scheletriche.

- Ruolo della professione medica nella società.

- Uso responsabile dell'autorità e del potere.

- Mobilitare le risorse in base alle esigenze.

- Adattare la pratica, la gestione e l'educazione alle esigenze dell'individuo.

- Diritti dei pazienti a cure efficaci e sicure.

- Principi di politica sanitaria e loro implicazioni. 
- Interazioni con altre figure e competenze descritte in questo documento.

- Struttura e funzionamento dei sistemi sanitari e di sicurezza sociale.

- Ripartizione delle risorse nei settori della sanità e nei sistemi di sicurezza sociale.

\section{Competenze chiave}

Il reumatologo deve essere in grado di...

1. Rispondere alle esigenze di salute del paziente come parte integrante dell'assistenza

2. Rispondere ai bisogni di salute della comunità

3. Individuare i fattori determinanti per la salute della popolazione

4. Promuovere la salute dei singoli pazienti, della comunità e della popolazione.

\section{Esigenze specifiche del processo formativo}

Queste competenze non possono essere completamente acquisite durante la formazione specialistica, ma saranno sviluppate e maturate attraverso lo sviluppo professionale continuo. I programmi di Specializzazione, tuttavia, devono fissare le condizioni di base per favorire questo processo e verificare che esse siano state acquisite.

Al completamento della formazione, il reumatologo deve essere in grado di dimostrare l'acquisizione delle seguenti nozioni, abilità e attitudini specifiche: 1. Rispondere alle esigenze di salute ed ai problemi del singolo paziente come parte integrante dell'attività di assistenza.

1.1. Identificare i bisogni di salute del singolo paziente.

1.2. Identificare e usare tutte le opportunità per la difesa, la promozione della salute e la prevenzione delle malattie con il singolo paziente e con chi lo assiste.

1.3. Conoscere i regolamenti della sanità e della sicurezza sociale.

1.4. Essere pronto a fornire consulenza in contenziosi medico-legali tra pazienti e compagnie di assicurazione o in casi di errori professionali.

2. Rispondere ai bisogni di salute della comunità. 2.1. Descrivere gli aspetti sociali e sanitari delle comunità in cui si opera.

2.2. Identificare le possibilità di difesa e promozione della salute e di prevenzione della malattia nella comunità in cui si opera, e prendere misure appropriate.

2.3. Valutare la possibilità di interessi contrastanti tra la comunità in cui si opera ed il resto della popolazione.
3. Individuare i fattori determinanti della salute per le popolazioni in cui si opera:

3.1. Individuare i fattori determinanti della salute della popolazione, tra cui gli ostacoli ad accedere alle cure.

3.2. Individuare I gruppi sociali vulnerabili o emarginati all'interno della collettività e le opportunità per migliorare la loro condizione.

4. Promuovere la salute dei singoli pazienti, delle comunità e della popolazione:

4.1. Descrivere e sostenere un approccio per attuare un cambiamento nella gestione delle risorse sanitarie della popolazione.

4.2. Descrivere in che modo gli effetti della politica pubblica influiscono sulla salute generale.

4.3. Identificare i punti di potenziale influenza personale nel sistema sanitario.

4.4. Dimostrare di possedere i principi dell'etica professionale relative alla difesa della salute, all'altruismo, alle questioni sociali, alla giustizia, all'integrità e all'idealismo.

4.5. Individuare la possibilità di un conflitto tra il ruolo di difensore della salute per il paziente o per la comunità e quello di dirigente del servizio sanitario.

4.6. Descrivere il ruolo della professione medica nel sostegno della salute e della sicurezza dei pazienti.

\section{Metodi di insegnamento ed apprendimento}

I metodi e le risorse che possono contribuire alla acquisizione di tali competenze includono, tra l'altro:

- Esperienze di apprendimento in Dipartimenti che sono eccellenza nel proprio campo.

- Discussioni di gruppo basate su argomenti specifici.

- Corsi dedicati all'etica e alla difesa della salute

- Assegnazione di ruolo di difensore.

- Lavorare con le associazioni di pazienti e di altri gruppi coinvolti in tale attività.

\section{Metodi di valutazione}

- Cartelle cliniche.

- Valutazione a $360^{\circ}$.

- Relazioni scritte.

- Discussioni su argomenti specifici.

\section{PER APPROFONDIMENTI}

1. European Board of Rheumatology Educational Guide (http://www.uemsrheumatology.net/). 


\section{LO STUDIOSO}

\section{Definizione}

Quale Studioso, il reumatologo dovrebbe svolgere un'attività continua di ricerca al fine di migliorare il proprio patrimonio di competenze e, nello stesso tempo, essere coinvolto nella produzione, nella diffusione e nell'applicazione di nuove conoscenze nel proprio campo di interesse.

\section{Descrizione}

Quale medico, il reumatologo riconosce la necessità di impegnarsi in attività di ricerca al fine di migliorare le proprie conoscenze, contribuire alla creazione, diffusione, applicazione e traduzione in pratica di nuove acquisizioni. Egli, inoltre, riconosce e svolge il ruolo di educatore (modello di riferimento), promuovendo la formazione di studenti, pazienti, colleghi e di altre figure professionali coinvolte nell'assistenza del paziente e della comunità.

\section{Elementi}

- Apprendimento continuo, per migliorare le competenze ed essere attendibili.

- Analisi di tutti gli aspetti della pratica clinica.

- Autovalutazione.

- Valutazione critica di evidenze, direttive e raccomandazioni.

- Medicina basata sull'evidenza.

- Principi di insegnamento e apprendimento.

- Ruolo di riferimento.

- Fornire riscontri.

- Attività di mentore.

- Valutazione degli studenti.

- Principi etici di autorità, riservatezza e dei confini del rapporto insegnante-studente.

- Principi della ricerca scientifica.

- Principi etici della divulgazione: conflitto di interessi, rapporti con le industrie.

\section{Competenze chiave}

Il reumatologo deve essere in grado di...

1. Dimostrare le competenze e le attitudini necessarie per migliorare la qualità della propria prestazione professionale attraverso l'apprendimento continuo e l'autovalutazione.

2. Applicare i principi della medicina basata sulle evidenze nella pratica clinica.

3. Facilitare l'acquisizione di conoscenze da parte di pazienti, familiari, studenti, specializzandi, dirigenti sanitari e di componenti della società a seconda dei casi.
4. Contribuire alla produzione, diffusione, applicazione, e trasferimento nella pratica di nuove conoscenze teoriche e pratiche, in particolare nel campo della reumatologia.

5. Interagire con l'industria e con altri interlocutori commerciali, con la dovuta considerazione dei rischi e tenendo conto dei conflitti di interesse.

\section{Esigenze specifiche del processo formativo}

Al completamento del processo formativo, il reumatologo deve dimostrare di possedere le seguenti nozioni, abilità e attitudini.

1. Dimostrare le competenze e le attitudini necessarie per migliorare la qualità della propria prestazione professionale attraverso l'apprendimento continuo e l'autovalutazione.

1.1. Conoscere i principi e le strategie utili a sviluppare ed attuare un programma continuo di crescita sul piano professionale.

1.2. Identificare le proprie lacune culturali e le proprie necessità di apprendimento.

1.3. Dimostrare l'acquisizione di metodi di autovalutazione e di criteri di analisi dei risultati.

1.4. Porre quesiti appropriate, accedere alle informazioni ed interpretare le evidenze.

1.5. Integrare nuove Informazioni nella pratica clinica.

1.6. Conoscere i principi di qualità nella gestione. 2. Applicare i principi della medicina basata sulle evidenze.

2.1. Conoscere i principi della medicina basata sulle evidenze.

2.2. Saper ricercare, identificare e valutare in modo critico evidenze (in un contesto di evidence-based medicine), al fine di poter affrontare qualsiasi tipo di problema clinico.

2.3. Integrare la suddetta valutazione critica nella pratica clinica, in particolare per quanto riguarda il rapporto rischio-beneficio.

3. Facilitare l'apprendimento da parte di pazienti, familiari, studenti, specializzandi, dirigenti medici e altri componenti della società a seconda dei casi. 3.1. Conoscere i principi dell'insegnamento e dell'apprendimento rilevanti per l'istruzione dei medici.

3.2. Individuare in modo collaborativo le esigenze di apprendimento di altri e gli obiettivi attesi.

3.3. Selezionare approcci didattici efficaci e contenuti tesi a facilitare l'apprendimento.

3.4. Dimostrare la capacità di comunicare efficacemente e di insegnare argomenti di Reumatologia ad altri operatori sanitari e ad esponenti del pubblico. 
3.5. Fornire un riscontro efficace.

3.6. Conoscere i principi di etica dell'insegnamento.

4. Contribuire allo sviluppo, alla diffusione e alla traduzione nella pratica clinica di nuove conoscenze, in particolare nel campo della reumatologia.

4.1. Conoscere i principi della ricerca e dell'indagine scientifica.

4.2. Conoscere i principi di etica della ricerca

4.3. Elaborare domande pertinenti e adeguate su temi di ricerca.

4.4. Eseguire una ricerca sistematica della letteratura.

4.5. Dimostrare una capacità di analisi critica dei metodi di ricerca appropriati ad affrontare un qualsiasi tipo di problema clinico.

4.6. Dimostrare di possedere la capacità di trasmettere i risultati di uno studio.

\section{Metodi di insegnamento e di apprendimento}

I metodi e le risorse che possono contribuire alla acquisizione di tali competenze includono, tra l'altro:

- Auto-apprendimento:

- Letture indipendenti: trattati e manuali, riviste, internet.

- Corsi dedicati:

- Medicina basata sull'evidenza.

- Metodologia della ricerca.

- Valutazione di risultati.

- Corsi di insegnamento rivolti a docenti (Teach the Teachers).

- Altri corsi.

- Esperienza in un ambiente supervisionato:

- Discussioni di gruppo guidate da docenti tutori

- Modulazione dell'attività da parte di docenti/tutori.

- Discussioni basate su casi interattivi.

- Partecipazione a verifiche individuali o di gruppo e ad altri progetti.

- Rivalutazione sistematica delle cartelle dei propri pazienti.

- Preparazione e presentazione della casistica.

- Progetti di ricerca.

- Insegnamento.

- Preparazione e presentazione di recensioni basate sulla Medicina basata sull'evidenza.

- Presentazioni a colleghi e ad un pubblico di profani.

\section{Metodi di valutazione}

I possibili metodi di valutazione di tali competenze comprendono:
- Valutazione periodica del livello di formazione con riscontri.

- Archivio di cartelle cliniche

- Valutazione a $360^{\circ}$.

- Valutazioni delle prestazioni in materia di ciascuna competenza specifica, a seguito di una valutazione condotta in maniera strutturata e con criteri predefiniti.

\section{PER APPROFONDIMENTI}

1. Errore. Riferimento a collegamento ipertestuale non valido. European Board of Rheumatology Educational Guide (http://www.uemsrheumatology.net/).

\section{IL PROFESSIONISTA}

\section{Definizione}

In qualità di Professionista, il reumatologo è impegnato alla promozione della salute e del benessere degli individui e della società con un'attività ispirata a principi di etica professionale e caratterizzata da elevati standard di comportamento personale.

\section{Descrizione}

Il reumatologo, come ogni medico, svolge un ruolo unico di professionista dedito alla salute e alla cura degli altri. Tale lavoro richiede la padronanza di un insieme complesso di conoscenze e competenze, così come della (ndr cosiddetta) arte della medicina. Quale Professionista, il Reumatologo deve svolgere la propria attività in aderenza a codici etici, e con competenze cliniche, che comprendono atteggiamenti e comportamenti adeguati, altruismo, e promozione del benessere pubblico. Questi aspetti costituiscono la base di un contratto tra il medico e la società. La società, in cambio, concede ai medici il privilegio di assumere un ruolo di riferimento, avendo essi responsabilità nei confronti dei propri pazienti.

\section{Elementi}

- Altruismo e empatia.

- Integrità e onestà.

- Capacità di comprendere e curare.

- Morale e codici di comportamento.

- Responsabilità verso la società.

- Responsabilità verso la professione, compresi gli obblighi di revisione tra pari.

- Responsabilità verso se stessi, compresa l'assistenza personale al fine di servire gli altri. 
- Impegno per l'eccellenza nella pratica clinica e padronanza della disciplin.

- Impegno per la promozione del bene pubblico nel settore sanitario.

- Responsabilità nei rapporti con l'ordine professionale.

- Impegno per mantenere alti standard professionali.

- Principi bioetici.

- Strutture medico-legali che disciplinano la pratica.

- Autocoscienza.

- Pratiche sostenibili e salute dei medici.

- Autovalutazione.

- Notificazione di errori o di eventi avversi.

\section{Competenze chiave}

In qualità di Professionista, il reumatologo deve:

1. Dimostrare un impegno per i propri pazienti, per la professione, e per la società attraverso una pratica etica.

2. Dimostrare un impegno per i propri pazienti, la professione e la società attraverso la partecipazione alla regolamentazione della propria professione. 3. Dimostrare un impegno per la salute dei medici e per pratiche sostenibili.

\section{Esigenze specifiche del processo formativo}

Queste competenze si sviluppano e maturano attraverso il continuo impegno professionale.

I programmi di specializzazione, tuttavia, devono includere le norme appropriate per promuovere comportamenti che assicurino un impegno permanente per mantenere i propri principi morali.

\section{Al completamento della formazione il reumatolo- go deve essere in grado di:}

1. Dimostrare impegno per i propri pazienti, per la professione, e per la società con un'attività etica.

1.1. Mostrare comportamenti professionali adeguati nella pratica clinica, informati a principi di onestà, integrità, impegno, compassione, rispetto e altruismo.

1.2. Dimostrare impegno a fornire una cura di massima qualità e a sviluppare competenze.

1.3. Dimostrare di rispondere alle esigenze e agli interessi dei pazienti, mettendo da parte l'interesse personale.

1.4. Dimostrare la capacità di rendere i propri pazienti liberi di scegliere la terapia dopo aver delineato tutte le opzioni ed i relativi rischi.

1.5. Fornire in modo comprensibile gli elementi chiave tesi ad ottenere il consenso informato e ri- chiederlo per interventi terapeutici e per attività di ricerca clinica.

1.6. Riconoscere l'esistenza di problemi etici nella pratica clinica, affrontarli adeguatamente ed essere sempre consapevoli di potenziali conflitti di interesse.

1.7. Gestire correttamente conflitti di interesse, con particolare attenzione ai rapporti con l'industria farmaceutica.

1.8. Conoscere i principi ei limiti della gestione dei dati riservati dei pazienti definiti dalle norme della pratica professionale e da leggi.

1.9. Mantenere rapporti adeguati con i pazienti.

2. Dimostrare un impegno per i propri pazienti, la professione e la società attraverso l'aderenza a codici di regolamento della propria professione.

2.1. Conoscere i codici professionali, legali ed etici che regolano la pratica clinica.

2.2. Adempiere agli obblighi normativi e giuridici richiesti dalla prassi attuale.

2.3. Essere responsabile nei confronti di organismi di regolamentazione professionale.

2.4. Identificare e reagire a comportamenti poco professionali di altri nella pratica clinica.

2.5. Partecipare ed assistere a valutazioni di comportamenti professionali.

3. Dimostrare un impegno per la salute propria e per le pratiche sostenibili.

3.1 Equilibrio personale e professionale al fine di garantire la salute personale e pratiche sostenibili.

3.2 Sforzarsi di incrementare la propria consapevolezza e la propria intuizione personale e professionale.

3.3 Riconoscere il ruolo di altri professionisti in caso di necessità e comportarsi di conseguenza in modo efficace.

\section{Metodi di insegnamento ed apprendimento}

- Esperienze in Dipartimenti esempi di buona pratica clinica costituiscono più efficace per promuovere queste competenze.

- Capacità di assumere un ruolo di modello e di tutor.

- Valutazione periodica del livello di formazione.

- Partecipazione ad attività professionalizzanti. Gli specializzandi devono avere garantita la possibilità di partecipare a servizi per la comunità, ad organizzazioni professionali e ad attività di associazioni istituzionali.

- Didattica: convegni, conferenze, dibattiti dedicati ad argomenti sulla professionalità.

- Discussioni di gruppo: Discussioni su casi clini- 
ci o riunioni di Journal club che forniscano l'opportunità di confronto tra docenti e allievi.

- Letture Indipendenti: pubblicazioni sottoposte al vaglio di revisori e pubblicazioni sulla professione di organizzazioni specializzate.

\section{Metodi di valutazione}

- Valutazione delle prestazioni - per quanto riguarda la dimostrazione di comportamenti professionali- da parte dei docenti/tutori.

- Valutazioni a 360 gradi per quanto riguarda gli atteggiamenti e i comportamenti professionali. Gli specializzandi possono anche compilare auto-valutazioni in materia di professionalità.

- Archivio delle cartelle - incluse annotazioni su questioni come pazienti difficili, conflitti di interesse, e ostacoli a fornire la cura giusta.

- Valutazione dei pazienti - con particolare attenzione agli elementi che riguardano specificamente la professionalità dello specializzando.

\section{PER APPROFONDIMENTI}

1. Rothman DJ, Medical professionalism - focusing on the real issues, N Engl J Med 2000; 342: 1284-6.

2. Klein EJ, Jackson JC, Kratz L, Marcuse EK, McPhillips HA, Shugerman RP, Watkins S, Stapleton FB. Teaching professionalism to residents, Acad Med 2003; 78: 26-34.

3. Hatem CJ. Teaching approaches that reflect and promote professionalism. Acad Med 2003; 78: 709-13.

4. ABIM Medical Professionalism in the new millenium: A physician charter. Ann Internal Med 2002; 136: 243-6.

5. Blank L. Medical professionalism in the new millennium. A physician charter 15 months later. Ann Internal Medicine 2003; 138: 839-41.
6. Steinert Y, Cruess S, Cruess R, Snell L, Faculty development for teaching and evaluating professionalism: from programme design to curriculum change. Med Educ 2005; 39: 127-36.

7. McCormick BB, Tomlinson G, Brill-Edwards P, Detsky AS. Effect of restricting contact between pharmaceutical company representatives and internal medicine residents on post-training attitudes and behavior. JAMA 2001; 286: 1994-9.

8. Kuczewski M. Fostering professionalism: the loyola model. Cambridge Quarterly of Healthcare Ethics 2003; 12: 161-6.

9. Branch W. Feedback and reflection: teaching methods for clinical settings. Academic Medicine 2002; 77: 1185-8.

10. Siegler M. Training doctors for professionalism: lessons learned from teaching clinical medical ethics. Mount Sinai Journal of Medicine 2002; 69: 404-9.

11. "Advancing Education in Professionalism". An educational resource developed by the ACGME to aid program directors. http://www.acgme.org/outcome/implement/Profm_resource.pdf

12. The ACGME also has a comprehensive list of professionalism references available at http://www. acgme.org/outcome/comp/refProf1.asp

13. The ACGME provides several assessment tools for the evaluation of professionalism: http://www. acgme.org/outcome/assess/profIndex.asp

14. NBME Embedding Professionalism in Medical Education: Assessment as a tool for Implementation 2002.http://www.nbme.org/PDF/NBME_AAMC_P rofessReport.pdf

15. NBME Behaviors of Professionalism http://ci.nbme. org/professionalism/

16. The American Medical Association Ethics Publication "Virtual Mentor" found at www.virtualmentor.org.

17. American Medical Association. "Embedding Professionalism in Medical Education: Assessment as a tool for implementation". http://www.nbme.org/ PDF/NBME_AAMC_ProfessReport.pdf 